\title{
Time-Optimal Control of a Small Mass Point without Environmental Resistance
}

\author{
A. R. Danilin ${ }^{a, b}$ and O. O. Kovrizhnykh ${ }^{a, b}$ \\ Presented by Academician A.M. Il'in January 28, 2013
}

Received February 11, 2013

DOI: $10.1134 / \mathrm{S} 1064562413040364$

We study the problem of the time-optimal control of a small mass point without environmental resistance. The control function in the problem is a magnitude-bounded force. The time required for transferring the point from one given state to another is used as a control criterion. It is shown that the optimal time and the optimal control have complicated asymptotic expansions even in the situation of general position.

1. In the study of various aspects of optimal control problems [1-3] for systems with fast and slow variables [4-8, 11] of the form

$$
\begin{gathered}
\dot{x}=A_{1} x+A_{2} y+B_{1} u, \quad x \in \mathbb{R}^{n}, \quad y \in \mathbb{R}^{m}, \\
\varepsilon \dot{y}=A_{3} x+A_{4} y+B_{2} u, \quad u \in \mathbb{R}^{k}
\end{gathered}
$$

an important role is played by the following condition.

Condition I. All the eigenvalues of the matrix $A_{4}$ have negative real parts.

However, there are problems with a meaningful mechanical interpretation in which Condition $I$ is not satisfied. An example is the following time-optimal control problem in the class of piecewise continuous controls:

$$
\begin{gathered}
\dot{x}=y, \quad x, y \in \mathbb{R}^{2}, \\
\varepsilon \dot{y}=u, \quad u \in \mathbb{R}^{2}, \quad\|u(t)\| \leq 1, \\
x(0)=x_{0} \neq 0, \quad y(0)=y_{0}, \quad 0<\varepsilon \ll 1, \\
x\left(T_{\varepsilon}\right)=0, \quad y\left(T_{\varepsilon}\right)=0, \quad T_{\varepsilon} \rightarrow \min .
\end{gathered}
$$

It can be interpreted as follows. Given a point particle of small mass $\varepsilon>0$ moving in a plane under the influence of a bounded control force $u(t)$ lying in this plane, the goal is the minimum-time transfer of the particle

\footnotetext{
${ }^{a}$ Krasovskii Institute of Mathematics and Mechanics, Ural Branch, Russian Academy of Sciences, ul. S. Kovalevskoi 16, Yekaterinburg, 620219 Russia

${ }^{b}$ Ural Federal University,

ul. Mira 19, Yekaterinburg, 620002 Russia

e-mail:dar@imm.uran.ru,koo@imm.uran.ru
}

from the given state defined by the position $\left(x_{0}\right)$ and the velocity $\left(y_{0}\right)$ to the origin, where it stops.

Here and below, $\|\cdot\|$ is the Euclidean norm and $\langle\cdot, \cdot\rangle$ is the inner product in the considered finitedimensional spaces.

The basic difference of this problem from previously considered ones is that $A_{4}=0$. As a result, there is no reduced system and, hence, it is unclear how the reduced problem for (2) looks like (and whether it exists at all).

Note that time-optimal control problems for completely controllable systems of more general form

$$
\begin{gathered}
\dot{x}=A y, \quad x, y \in \mathbb{R}^{n}, \\
\varepsilon \dot{y}=B u, \quad u \in \mathbb{R}^{m}, \quad\|u(t)\| \leq 1, \\
x(0)=x_{0}, \quad y(0)=y_{0}, \quad 0<\varepsilon \ll 1, \\
x\left(T_{\varepsilon}\right)=0, \quad y\left(T_{\varepsilon}\right)=0, \quad T_{\varepsilon} \rightarrow \min
\end{gathered}
$$

can be reduced to problem (2) by changing variables.

The following result is straightforward.

Proposition 1. For all sufficiently small \& problem (2) is solvable, and $T_{\varepsilon} \rightarrow 0$ as $\varepsilon \rightarrow 0$.

As will be shown later, $T_{\varepsilon}=O(\sqrt{\varepsilon})$ as $\varepsilon \rightarrow 0$, and the characteristic scaled time in problem (2) is $\frac{t}{\sqrt{\varepsilon}}$ rather than $\frac{t}{\varepsilon}$, which occurs in problems satisfying Condition I.

The goal of this study is to construct complete asymptotic expansions in powers of the small parameter $\varepsilon$ for the optimal time and the optimal control in problem (2). To this end, we use the methods developed in $[9,10]$.

It should be stressed that, even in the situation of general position, the asymptotic expansions of these quantities have a complicated character similar to that of the asymptotics obtained in $[9,10]$. 
2. In problem (2) we pass to the new time $\tau:=\frac{t}{\sqrt{\varepsilon}}$ and denote $x(\sqrt{\varepsilon} \tau), \sqrt{\varepsilon} y(\sqrt{\varepsilon} \tau), u(\sqrt{\varepsilon} \tau)$, and $\frac{T_{\varepsilon}}{\sqrt{\varepsilon}}$ by $X(\tau), Y(\tau), U(\tau)$, and $\theta_{\varepsilon}$, respectively. Then problem (2) becomes

$$
\begin{gathered}
X^{\prime}=Y, \quad X, Y \in \mathbb{R}^{2}, \\
Y^{\prime}=U, \quad U \in \mathbb{R}^{2}, \quad\|U(\tau)\| \leq 1, \\
X(0)=x_{0}, \quad Y(0)=\sqrt{\varepsilon} y_{0}, \quad 0<\varepsilon \ll 1, \\
X\left(\theta_{\varepsilon}\right)=0, \quad Y\left(\theta_{\varepsilon}\right)=0, \quad \theta_{\varepsilon} \rightarrow \text { min. }
\end{gathered}
$$

By Proposition 1, new problem (3) is solvable at all sufficiently small $\varepsilon$. Moreover, it is well known [1] that problem (3) is also solvable at $\varepsilon=0$. Note that, for $\varepsilon=0$, the optimal control is discontinuous.

Theorem 1. Problem (3) is solvable at all small $\varepsilon \geq 0$ and

$$
\theta_{\varepsilon} \rightarrow \theta_{0}=2 \sqrt{\left\|x_{0}\right\|} \text { as } \varepsilon \rightarrow 0 .
$$

3. By Pontryagin's maximum principle [1, 3], which is a necessary and sufficient optimality condition in the case under consideration, the optimal control in problem (3) is given by

$$
U_{\text {opt }}(\tau)=\frac{-\tau l_{1, \varepsilon}+l_{2, \varepsilon}}{\left\|-\tau l_{1, \varepsilon}+l_{2, \varepsilon}\right\|}, \quad l_{1, \varepsilon}, l_{2, \varepsilon} \in \mathbb{R}^{2},
$$

and this representation is unique up to the normalization of the vector $\left(l_{1, \varepsilon}, l_{2, \varepsilon}\right)$.

For problem (3) with $\varepsilon=0$, these vectors with the normalization condition $\left\|l_{1,0}\right\|=1$ have the form $l_{2,0}=$ $\lambda_{0} l_{1,0}$, where $\lambda_{0}=\sqrt{\left\|x_{0}\right\|}$, and $l_{1,0}=-\frac{x_{0}}{\left\|x_{0}\right\|}$.

By virtue of the Cauchy formula, a control of form (4) is optimal for problem (3) if and only if

$$
-\left(\begin{array}{c}
x_{0} \\
\sqrt{\varepsilon} y_{0}
\end{array}\right)=\int_{0}^{\theta_{\varepsilon}}\left(\begin{array}{c}
\tau^{2} l_{1, \varepsilon}-\tau l_{2, \varepsilon} \\
-\tau l_{1, \varepsilon}+l_{2, \varepsilon}
\end{array}\right) \frac{d \tau}{\left\|-\tau l_{1, \varepsilon}+l_{2, \varepsilon}\right\|} .
$$

In what follows, we assume that the initial vectors in problem (2) are not parallel:

$$
x_{0} \nVdash y_{0} \text {. }
$$

Condition (6) corresponds to the situation of general position (i.e., it remains valid under small variations in $x_{0}$ and $y_{0}$ )

A solution of Eq. (5) is sought in the form

$$
\begin{gathered}
l_{1, \varepsilon}=l_{1,0}+r, \quad\left\|l_{1, \varepsilon}\right\|=1, \\
l_{2, \varepsilon}=\left(\lambda_{0}+v\right) l_{1, \varepsilon}+\tilde{l}_{2, \varepsilon}, \quad \tilde{l}_{2, \varepsilon} \perp l_{1, \varepsilon}, \\
\beta=\left\|\tilde{l}_{2, \varepsilon}\right\|, \quad \theta_{\varepsilon}=\theta_{0}+\vartheta,
\end{gathered}
$$

where all the quantities introduced are small.

Evaluating the integral in (5) and taking into account (7), we obtain the system of equations

$$
\begin{gathered}
-x_{0}=l_{1, \varepsilon}\left(\frac{1}{2} F_{2}(\vartheta, v, \beta)\right. \\
\left.-\frac{1}{2} \beta^{2} F_{0}(\vartheta, v, \beta)+\left(\lambda_{0}+v\right) F_{1}(\vartheta, v, \beta)\right) \\
-\tilde{l}_{2, \varepsilon}\left(F_{1}(\vartheta, v, \beta)+\lambda F_{0}(\vartheta, v, \beta)\right), \\
-\sqrt{\varepsilon} y_{0}=-l_{1, \varepsilon} F_{1}(\vartheta, v, \beta)+\tilde{l}_{2, \varepsilon} F_{0}(\vartheta, v, \beta), \\
\left\|l_{1, \varepsilon}\right\|=1, \quad\left\langle\tilde{l}_{2, \varepsilon}, l_{1, \varepsilon}\right\rangle=0, \\
\beta=\left\|\tilde{l}_{2, \varepsilon}\right\|,
\end{gathered}
$$

where

$$
\begin{gathered}
F_{0}(\vartheta, v, \beta) \\
=\ln \left(\frac{\theta_{0} / 2+\vartheta-v+\sqrt{\left(\theta_{0} / 2+\vartheta-v\right)^{2}+\beta^{2}}}{-\theta_{0} / 2-v+\sqrt{\left(\theta_{0} / 2+v\right)^{2}+\beta^{2}}}\right), \\
=\frac{\left(\theta_{0} / 2+\vartheta-v\right)^{2}-\left(\theta_{0} / 2+v\right)^{2}}{\sqrt{\left(\theta_{0} / 2+\vartheta-v\right)^{2}+\beta^{2}}+\sqrt{\left(\theta_{0} / 2+v\right)^{2}+\beta^{2}}}, \\
F_{2}(\vartheta, v, \beta) \\
=\left(\theta_{0} / 2+\vartheta-v\right) \sqrt{\left(\theta_{0} / 2+\vartheta-v\right)^{2}+\beta^{2}} \\
+\left(\theta_{0} / 2+v\right) \sqrt{\left(\theta_{0} / 2+v\right)^{2}+\beta^{2}} .
\end{gathered}
$$

If $r, v, \beta$, and $\vartheta$ are all sufficiently small, then the functions $F_{0}, F_{1}$, and $F_{2}$ can be represented as

$$
\begin{gathered}
F_{0}(\vartheta, v, \beta)=2 \ln \frac{\theta_{0}}{\beta}+\frac{2 \vartheta}{\theta_{0}}+\frac{2 \beta^{2}}{\theta_{0}^{2}}-\frac{2}{\theta_{0}^{2}}\left(v^{2}+(\vartheta-v)^{2}\right) \\
+\mathscr{F}_{0,3}(\vartheta, v, \beta), \\
F_{1}(\vartheta, v, \beta)=\vartheta-2 v+\mathscr{F}_{1,3}(\vartheta, v, \beta), \\
F_{2}(\vartheta, v, \beta)=\frac{\theta_{0}^{2}}{2}+\theta_{0} \vartheta+\beta^{2}+(\vartheta-v)^{2} \\
+v^{2}+\mathscr{F}_{2,3}(\vartheta, v, \beta),
\end{gathered}
$$

where $\mathscr{F}_{i, 3}(\vartheta, \nu, \beta)$ are converging power series beginning with the third power of their arguments with known coefficients.

Solving the first approximation system for (8) in view of expansions (9) yields

$$
\begin{gathered}
\vartheta_{1}=-\sqrt{\varepsilon}\left\langle y_{0}, l_{1,0}\right\rangle, \quad v_{1}=\vartheta_{1}, \\
r_{1}=-\frac{2}{\theta_{0}} \sqrt{\varepsilon} y_{0}+\frac{2}{\theta_{0}} \sqrt{\varepsilon} l_{1,0}\left\langle y_{0}, l_{1,0}\right\rangle .
\end{gathered}
$$

Moreover, $\tilde{l}_{2,1}$ satisfies the equation

$$
2 \tilde{l}_{2,1} \ln \frac{\theta_{0}}{\beta_{1}}=\sqrt{\varepsilon}\left(l_{1,0}\left\langle y_{0}, l_{1,0}\right\rangle-y_{0}\right)=: \sqrt{\varepsilon} \vee_{0} \stackrel{(6)}{\neq} 0 .
$$

DOKLADY MATHEMATICS Vol. $88 \quad$ No. $1 \quad 2013$ 
Thus, $\beta_{1}=\left\|\tilde{l}_{2,1}\right\|$ satisfies the equation $2 \beta_{1} \ln \frac{\theta_{0}}{\beta_{1}}=$ $\sqrt{\varepsilon}\left\|v_{0}\right\|$. From this, denoting the inverse of the function $\delta \ln \frac{1}{\delta}$ by $W_{0}(\cdot)$, we obtain, for small $\delta$,

$$
\begin{aligned}
\beta_{1}=W(\varepsilon) & :=\theta_{0} W_{0}\left(\frac{\sqrt{\varepsilon}\left\|x_{0}\right\|}{2 \theta_{0}}\right)=o(\sqrt{\varepsilon}), \\
\varepsilon & \rightarrow 0(\text { see }[9,10])
\end{aligned}
$$

Next, asymptotic expansions for all the indicated quantities are derived following the standard technique (see $[9,10])$. They are similar in form to the expansions obtained in $[9,10]$. Thus, the following result holds.

Theorem 2. Under condition (6), the optimal time $T_{\varepsilon}$ and the components of vectors $l_{1, \varepsilon}$ and $l_{2, \varepsilon}$ can be expanded in asymptotic series of the form $\sum_{k=0}^{\infty} R_{k}\left(\varepsilon, W(\varepsilon), \ln \frac{1}{W(\varepsilon)}\right)$, where $R_{k}(\cdot)$ are rational functions of their arguments and $R_{k}\left(\varepsilon, W(\varepsilon), \ln \frac{1}{W(\varepsilon)}\right)=O\left(\varepsilon^{k / 2}\right)$.

More specifically,

$$
\begin{gathered}
\theta_{\varepsilon}=\theta_{0}-\sqrt{\varepsilon}\left\langle y_{0}, l_{1,0}\right\rangle-\frac{W^{2}(\varepsilon)}{\theta_{0}}+\sqrt{\varepsilon} W(\varepsilon) \frac{\left\|v_{0}\right\|}{\theta_{0}} \\
+P \varepsilon+O\left(\varepsilon^{2}\right), \\
l_{1, \varepsilon}=l_{1,0}+\frac{2 \sqrt{\varepsilon}}{\theta_{0}} v_{0}+O(\varepsilon), \\
l_{2, \varepsilon}=\frac{\theta_{0}}{2} l_{1,0}-\sqrt{\varepsilon} y_{0}+\frac{v_{0}}{\left\|v_{0}\right\|} W(\varepsilon)+O(\varepsilon), \quad \varepsilon \rightarrow 0,
\end{gathered}
$$

where $P$ is a given constant.

Thus, by virtue of formula (4), the asymptotic representation of the optimal control in problem (3) (and, hence, in problem (2)) has an irregular form up to $O(\varepsilon)$. For example, for $\left|-\tau+\frac{\theta_{0}}{2}\right| \gg \sqrt{\varepsilon}$, the optimal control has the asymptotic representation

$$
\begin{gathered}
U_{\mathrm{opt}}(\tau)=\operatorname{sgn}\left(-\tau+\frac{\theta_{0}}{2}\right) l_{1,0} \\
+\frac{V_{1} W(\varepsilon)-\tau \sqrt{\varepsilon} l_{1,1}-\sqrt{\varepsilon} y_{0}+\left\langle l_{1,0}, \tau \sqrt{\varepsilon} l_{1,1}+\sqrt{\varepsilon} y_{0}-v_{1} W(\varepsilon)\right\rangle l_{1,0}}{\left|-\tau+\frac{\theta_{0}}{2}\right|}+O(\varepsilon)
\end{gathered}
$$

as $\varepsilon \rightarrow 0$. Here, $l_{1,1}=\frac{2 v_{0}}{\theta_{0}}$ and $a v_{1}=\frac{v_{0}}{\left\|v_{0}\right\|}$.

\section{ACKNOWLEDGMENTS}

The work was supported in part by the Russian Foundation for Basic Research (project no. 11-01-00679) under the basic research program Fundamental Problems of Nonlinear Dynamics in Mathematics and Physics of the Presidium of the Russian Academy of Sciences and by the Ural Branch of the Russian Academy of Sciences (project no. 12-P-1-1009).

\section{REFERENCES}

1. L. S. Pontryagin, V. G. Boltyanskii, R. V. Gamkrelidze, and E. F. Mishchenko, The Mathematical Theory of Optimal Processes (Gordon and Breach, New York, 1986).

2. N. N. Krasovskii, Theory of Motion Control (Nauka, Moscow, 1968) [in Russian].
3. V. M. Alekseev, V. M. Tikhomirov, and S. V. Fomin, Optimal Control (Nauka, Moscow, 1979) [in Russian].

4. P. V. Kokotovic and A. H. Haddad, IEEE Trans. Autom. Control. 20 (1), 111-113 (1975).

5. A. B. Vasil'eva and M. G. Dmitriev, Mathematical Analysis: Advances in Science and Engineering (VINITI, Moscow, 1982) [in Russian].

6. A. L. Dontchev, Perturbations, Approximations, and Sensitivity Analysis of Optimal Control Systems (Heidelberg: Springer-Verlag, Berlin, 1983).

7. T. R. Gichev and A. L. Dontchev, Prikl. Mat. Mekh. 43 (3), 466-474 (1979).

8. A. I. Kalinin and K. V. Semenov, Comput. Math. Math. Phys. 44, 407-417 (2004).

9. A. R. Danilin and A. M. Il'in, Tekh. Kibern., No. 3, 96-103 (1994).

10. A. R. Danilin and A. M. Il'in, Fundam. Prikl. Mat. 4, 905-926 (1998).

11. A. R. Danilin and O. O. Kovrizhnykh, Tr. Inst. Mat. Mekh. 18 (2), 67-79 (2012).

Translated by I. Ruzanova 